\title{
Development of Antimicrobials against Escherichia coli - Environmental Microbiology meets Chemical Biotechnology
}

\section{Edward PC Lai* and Zafar Iqbal}

Department of Chemistry, Carleton University, 1125 Colonel by Drive, Ottawa, Ontario K1S 5B6, Canada

Escherichia coli (E. coli) is the commonest infecting uropathogen with its incidence climbing from $50 \%$ to $60 \%$ of Urinary Tract Infections (UTIs). A recent report has demonstrated a diverse antimicrobial-resistant E. coli population distributed over various areas (nasal, oral, rectal, abdomen and hindquarter) of a companion animal's body, thereby suggesting potential transfer of resistant micro flora to human hosts during contact. Epidemiological patterns in UTIs show large inter-regional variability, and rates of bacterial resistance are continually changing due to different regional antibiotic treatment regime [1]. E. coli is also one of the most important causes of nosocomial infections. In hospitals, trimethoprim or nitrofurantoin is usually recommended for empirical treatment of uncomplicated cystitis whilst parenteral cephalosporins, aminoglycosides, quinolones and coamoxyclav are reserved for complicated UTIs. Bloodstream Infections (BSIs) are often treated with fluoroquinolones and $\beta$-lactams (mainly gentamicin, ciprofloxacin and third-generation cephalosporins). The antimicrobial susceptibility pattern of uropathogenic E. coli strains (isolated from urine specimens) was determined using 13 common antibiotics [2]. The rates of antibiotic resistance of bio film producing E. coli were found to be $100 \%$ for chloramphenicol and amoxyclav (amoxicillin and clavulanic acid), 86\% for gentamicin and cefotaxime, $84 \%$ for ceftazidime, $83 \%$ for cotrimoxazole and piperacillin/ tazobactam, 75\% for tetracycline, and 70\% for amikacin. An ecological study investigating the correlation between the level of antimicrobial consumption and resistance, however, resistance to these compounds was reported with increasing frequency [3]. The dramatic rate increase in gentamicin resistance is of paramount concern. Neither ampicillin nor trimethoprim represent suitable empirical antimicrobials for UTI, and ciprofloxacin resistance renders it unsuitable empirical therapy for nosocomial UTIs. BSIs caused by 3 GC-resistant E. coli are associated with excess mortality and prolonged hospital stay, adding to the suffering of patients and posing a considerable burden on healthcare costs [4]. Global efforts to reduce the consumption of broad-spectrum antimicrobial agents are hence warranted. Fortunately, E. coli - induced UTIs have remained extremely sensitive to nitrofurantoin and the resistance rate has not changed significantly over the last decade.

Antimicrobial agent usage is common in animal agriculture for therapeutic and prophylactic purposes. Selective pressure exerted by these antimicrobials on soil bacteria could result in the selection of strains that are resistant due to chromosomal- or plasmid-derived genetic components [5]. Analysis of antibiotic resistance showed that $67 \%(n=29 / 43)$ of the enterotoxigenic E. coli strains were resistant to one or several of the antimicrobial agents tested. The presence of colonization factors was associated with antibiotic resistance [6]. Cephalosporinase producing E. coli has recently been detected in meat and animals [7]. Antimicrobial resistance was common among 95 strains of E. coli from pre-weaned dairy calves, occurring particularly in calves from herds experiencing calf diarrhea problems [8]. E. coli isolates from both dogs and cats exhibited resistance to all antimicrobials tested with the exception of amikacin, cephalothin and kanamycin. Resistance to ampicillin was the most prevalent resistance phenotype detected (dogs, 33/199; 17\%; and cats, 27/118; 23\%) [9]. A high prevalence of antimicrobial-resistant $E$. coli (producing extendedspectrum $\beta$-lactamase) can have significant health implications for the horse population [10], and methods to minimise their potential spread should be considered [11]. Antimicrobial Peptides (AMPs) have potential for use as alternatives to antibiotics in diets fed to weaned piglets; cecropin $\mathrm{AD}$ appeared to reduce diarrhoea incidence after $E$. coli challenge [12].

Development of antimicrobials against $E$. coli in water is another peaking research field. Previous literatures stated that optical density at $640 \mathrm{~nm}$ would be a good parameter to measure anti-bacterial activity against $E$. coli in water. Organic compounds could be a feasible approach towards killing E. coli in water. They concluded that $\% \mathrm{NH}_{2}$ was the most important factor for antibacterial activity of claimed chemicals. This specific OD would tell us whether the applied antimicrobials have any demonstrable bacteriostatic property towards E. coli. Addition of amino acid to Phosphatidyglycerol (PG) of the membrane is one of the mechanisms used by bacteria to lower the net charge of their cellular envelope, thereby decreasing its affinity for several antibacterial agents [13]. An appropriate antibacterial needs to be as strong as to be able to supersede this auto-induced power in bacteria against antibacterial compounds. AMPs with broad-spectrum activity, which can physically destroy the bacterial membrane through several targets to kill the microbes, may be considered a promising approach to overcome the problem. A rapid method, which coupled cell membrane affinity extraction with offline liquid chromatography time-of-flight mass spectrometry, was developed for screening and identifying antimicrobial peptides obtained by proteolytic enzyme hydrolysis [14]. A cationic antimicrobial peptide was successfully isolated, and antimicrobial assay indicated that it was active against $E$. coli ATCC 25922. Antimicrobial susceptibility was tested by determination of minimum inhibitory concentration, which was found to be $29 \pm 1 \mu \mathrm{g} /$ $\mathrm{mL}$ for the peptide, showing no increase in optical density at $600 \mathrm{~nm}$. This antimicrobial induced significant morphological alteration of the microbe surface by transmission electron microscopy, indicating strong membrane disruption. The antimicrobial activities of caseicin A and B AMPs were assessed against a selection of verocytotoxigenic

*Corresponding author: Edward PC Lai, Department of Chemistry, Carleton University, 1125 Colonel By Drive, Ottawa, Ontario K1S 5B6, Canada, E-mail: edward_lai@carleton.ca

Received May 17, 2012; Accepted May 19, 2012; Published May 21, 2012

Citation: Lai EPC, Iqbal Z (2012) Development of Antimicrobials against Escherichia coli - Environmental Microbiology meets Chemical Biotechnology. J Pet Environ Biotechnol 3:e106. doi:10.4172/2157-7463.1000e106

Copyright: ( 2012 Lai EPC, et al. This is an open-access article distributed under the terms of the Creative Commons Attribution License, which permits unrestricted use, distribution, and reproduction in any medium, provided the original author and source are credited. 
Escherichia coli strains. The mean number of E. coli surviving after exposure to $2 \mathrm{mg} / \mathrm{mL}$ caseicin A and B was reduced by 4.96 and 4.19 $\log _{10} \mathrm{cfu} / \mathrm{mL}$ compared to the respective controls [15].

Polydopamine (PDA) is well-known for its adhesive property in water [16]. One could use chitosan/chitin (which is an easily available hydrophilic polymer) in combination with PDA as a coating material on Magnetic Nanoparticles (MNPs) perhaps to simultaneous seize and kill $E$. coli in water. Chitosan, in its own right, can be used to enhance the antibacterial effect of PDA. This $\mathrm{N}$-acetyl glucosamine polymer can also be used to stabilize the MNPs@PDA particles [17]. Antibacterial activity of chitosan against E. coli was demonstrated previously [18]. Development of Molecularly Imprinted Polymer (MIP) to seize the bacteria from waters by the aid of external magnetic field would be a promising green strategy. Although imprinting of small organic and biomolecules is well achieved nowadays, large proteins, bacteria and viruses still present challenges due to their molecular size, complexity, conformational flexibility and solubility factors. Bacteria are not always compatible with mainstream MIP preparation in organic solvents. Highly cross-linked rigid networks of MIPs often result in low binding capacity and poor site accessibility due to slow diffusion of bacteria in the interior of three-dimensional polymeric matrices. The flexible structure and conformation of protein can be easily affected by the surrounding microenvironment of the imprinted cavity [19]. New functional monomers with good biocompatibility and multifunctional groups are a necessary requirement in order to eliminate these problems. PDA is a very good candidate in this regard as because the monomer DA is a small molecule mimic of proteins and it has plenty of non-covalent functionalities such as amino, imino, hydroxyl, catechol-containing groups as well as $\pi-\pi$ interactions. PDA forms a 2-dimensional fishnet over a solid surface (or around a solid particle). DA can create a 2-dimensional fishnet around any bacterium. Thus the DA could encapsulate bacteria or any other types of microorganisms in water. In addition, PDA (produced from oxidative polymerization of DA in water) may have bacteriostatic property due to its amino and imino functional groups. Therefore, the use of ammonia, an organic amine, or acryl amide (as a secondary functional monomer for the preparation of MIP) together with PDA would increase MIP's bacteriostatic property extensively. Thus, an appropriate method for simultaneous seizing and killing of bacteria in water could be developed. This research would emerge as one step forward in the field of environmental biotechnology.

Increasing resistance of all microbes is a growing worldwide problem. The increased use of antimicrobial agents in human medicine and food animal production is a significant factor in the emergence of antibiotic-resistant bacteria. Multiple antimicrobial resistances in $E$. coli and the direct relationship between antimicrobial agent uses over time have been extensively studied. The emergence and development of antimicrobial-resistant E. coli, as in other bacteria, may be a major public health problem. Particularly, extended-spectrum beta-lactamase producing E. coli pose real challenge to clinical microbiologists, clinicians, antibacterial researchers, veterinarians and food scientists [20]. More knowledge is required for prudent use of antimicrobials, and new development of alternative therapies against $E$. coli needs to be explored.

\section{References}

1. Cullen IM, Manecksha RP, McCullagh E, Ahmad S, O'Kelly F, et al. (2012) The changing pattern of antimicrobial resistance within 42, 033 Escherichia coli isolates from nosocomial, community and urology patient-specific urinary tract infections, Dublin, 1999-2009. BJU Int 109: 1198-1206.

2. Ponnusamya $P$, Natarajana $V$, Sevanan $M(2012)$ In vitro biofilm formation by uropathogenic Escherichia coli and their antimicrobial susceptibility pattern. Asian Pac J Trop Med 5: 210-213.

3. Skjøt-Rasmussen L, Olsen SS, Jensen US, Hammerum AM (2012) Increasing consumption of antimicrobial agents in Denmark parallels increasing resistance in Escherichia coli bloodstream isolates. Int J Antimicrob Agents.

4. de Kraker ME, Davey PG, Grundmann H, BURDEN study group (2011) Mortality and hospital stay associated with resistant Staphylococcus aureus and Escherichia coli bacteremia: estimating the burden of antibiotic resistance in Europe. PLoS Med 8: e1001104.

5. Jones SE, Burgos JM, Lutnesky MM, Sena JA, Kumar S, et al. (2010) Dairy farm age and resistance to antimicrobial agents in escherichia coli isolated from dairy topsoil. Curr Microbiol 62: 1139-1146.

6. Rodas C, Mamani R, Blanco J, Blanco JE, Wiklund G, et al. (2011) Enterotoxins, colonization factors, serotypes and antimicrobial resistance of enterotoxigenic Escherichia coli (ETEC) strains isolated from hospitalized children with diarrhea in Bolivia. Braz J Infect Dis 15: 132-137.

7. Agersø Y, Aarestrup FM, Pedersen K, Seyfarth AM, Struve T, et al. (2012) Prevalence of extended-spectrum cephalosporinase (ESC)-producing Escherichia coli in Danish slaughter pigs and retail meat identified by selective enrichment and association with cephalosporin usage. J Antimicrob Chemother 67: 582-588.

8. de Verdier K, Nyman A, Greko C, Bengtsson B (2012) Antimicrobial resistance and virulence factors in Escherichia coli from Swedish dairy calves. Act Vet Scand 54: 2

9. Davis JA, Jackson CR, Fedorka-Cray PJ, Barrett JB, Brousse JH, et al. (2011) Anatomical distribution and genetic relatedness of antimicrobial $\square$ resistant Escherichia coli from healthy companion animals. J Appl Microbiol 110: 597604.

10. Maddox TW, Clegg PD, Diggle PJ, Wedley AL, Dawson S, et al. (2012) Cross-sectional study of antimicrobial-resistant bacteria in horses. Part 1 Prevalence of antimicrobial-resistant Escherichia coli and methicillin-resistant Staphylococcus aureus. Equine Vet J 44: 289-296.

11. Johns I, Verheyen K, Good L, Rycroft A (2012) Antimicrobial resistance in faecal Escherichia coli isolates from horses treated with antimicrobials: A longitudinal study in hospitalised and non-hospitalised horses. Vet Microbiol.

12. Wu S, Zhang F, Huang Z, Liu H, Xie C, et al. (2012) Effects of the antimicrobial peptide cecropin $A D$ on performance and intestinal health in weaned piglets challenged with Escherichia coli. Peptides 35: 225-230.

13. Roy H (2009) Tuning the properties of the bacterial membrane with aminoacylated phosphatidylglycerol. IUBMB Life 61: 940-953.

14. Xiao J, Zhang H (2012) An Escherichia coli cell membrane chromatographyoffline LC-TOF-MS method for screening and identifying antimicrobial peptides from Jatropha curcas meal protein isolate hydrolysates. J Biomol Screen.

15. McDonnell MJ, Rivas L, Burgess CM, Fanning S, Duffy G (2012) Inhibition of verocytotoxigenic Escherichia coli by antimicrobial peptides caseicin $\mathrm{A}$ and $\mathrm{B}$ and the factors affecting their antimicrobial activities. Int J Food Microbiol 153 260-268.

16. Lee H, Rho J, Messersmith PB (2009) Facile conjugation of biomolecules onto surfaces via mussel adhesive protein inspired coatings. Adv Mater 21: 431-434.

17. Sureshkumar M, Lee CK (2011) Polydopamine coated magnetic-chitin (MCT) particles as a new matrix for enzyme immobilization. Carbohydr Polym 84: 775780

18. Xie Y, Liu X, Chen Q (2007) Synthesis and characterization of water-soluble chitosan derivate and its antibacterial activity. Carbohydr Polym 69: 142-147.

19. Ouyang R, Lei J, Ju H (2010) Artificial receptor-functionalized nanoshell: facile preparation, fast separation and specific protein recognition. Nanotechnology 21: 185502.

20. Arslan S, Eyi A (2011) Antimicrobial resistance and ESBL prevalence in Escherichia coli from retail meats. J Food Saf 31: 262-267. 